\title{
Oral health problems and quality of life
}

\author{
Sue Buckley and Ben Sacks
}

There is a higher incidence of oral health problems among individuals with Down syndrome, particulary after 10 years of age, indicating a need for better teaching of teeth brushing and more regular visits to the dentist. Do these oral health problems affect their quality of life?

This is the question addressed by Loureiro and her colleagues in Brazil ${ }^{[1]}$. A number of studies have indicated that children and adults with Down syndrome may experience higher rates of gum disease and its consequences than typically developing individuals. In this study of 93 children and young people (6 to 20 year olds), the researchers investigated their oral health by individual clinical examinations and also interviewed their mothers about the impact of oral health issues in their daily lives.

The clinical examinations included investigating the health of gums, and the state of the bones to which teeth are joined. Gum disease (gingivitis) can lead to sore gums, to the shrinking of gums away from the teeth and to loss of bone supporting teeth (periodontitis). All these indicators were measured and the degree of bone loss was rated as slight/moderate or severe defined by measurement of bone loss. Disease is caused by the build up of plaque and the amount of plaque present was also measured with a standard measure. The results of this part of the study showed that the incidence of plaque was high and that the oral hygiene of $68 \%$ of the individuals could be classified as bad or extremely bad. Bleeding on brushing or probing is an indication of gum disease and bleeding was reported for $91 \%$ of the individuals with Down syndrome. Some $30 \%$ of the group showed evidence of bone loss affecting the attachment of teeth to the bone.

The researchers then investigated the effects of the degree of oral health problems on various quality of life indicators including parents' perceptions of effects on speech, bad breath (halitosis), taste and discomfort when eating, and general daily life. From the quality of life questionnaire, they computed an impact score and report a significant correlation between the severity of gum and periodontal disease and quality of life.

In another recent study, Folakemi Ore$\mathrm{dugba}^{[2]}$ reports the findings of a study of the oral health and dental needs of individuals with Down syndrome from preschool to adult years in Nigeria. They compare their oral health with that of an age-matched comparison group of typically developing individuals. As a group, the individuals with Down syndrome have a higher level of oral health problems, but this only applies to those over 10 years of age, suggesting that while parents and carers are helping to clean teeth, oral hygiene may be satisfactory. Overall, more individuals with Down syndrome were in need of dental treatment.

These data clearly raise issues about the dental health care needs of individuals with Down syndrome as these figures are higher than would be expected in the general population. Interestingly, a recent study from Japan ${ }^{[3]}$ reports on the positive effects of dental visits and cleaning at 3-4 month intervals for a group of young adults with Down syndrome. They compared those who received this regular care with those who had not been seen for any dental or oral hygiene care for more than a year. The progress of periodontal disease was significantly less in the group with regular care.

Sue Buckley and Ben Sacks are at The Down Syndrome Educational Trust, Portsmouth, Hampshire, UK.e-mail: sue.buckley@downsed.org

doi:10.3104/updates.2049

\section{GLOSSARY}

Dental plaque is a film of mainly bacteria that builds up on teeth and is not easy to remove completely by tooth brushing. It can form a hard deposit between teeth and gums. The bacteria in plaque can, over time, lead to gingivitis and periodontal disease, damaging both teeth and gums.

Gingivitis (inflammation of the gums around the teeth) is a general term for diseases affecting the gingiva (gums).

The symptoms of gingivitis include:

- swollen gums

- gums that bleed easily

- receding gum line

Periodontitis in the mouth refers to progressive loss of bone around the teeth which may lead to loosening and eventual loss of teeth if untreated.

Individuals who have symptoms of gingivitis will benefit from having teeth and gums cleaned professionally every 3-4 months to prevent long term damage to them.

1. Loureiro ACA, Costa FO, da Costa JE. The impact of periodontal disease on the quality of life of individuals with Down syndrome. Down Syndrome Research and Practice. 2007;12(1):50-54.

2. Oredugba F. Oral health condition and treatment needs of a group of Nigerian individuals with Down syndrome. Down Syndrome Research and Practice. 2007; 12(1): 72-76.

3. Yoshihara T, Morinushi T, Kinjyo S, Yamasaki Y. Effect of periodic preventative care on the progression of periodontal disease in young adults with Down's syndrome. Journal of Clinical Periodontology, 2005;32:556-560. 